\title{
An Alternate Proof of the De Branges Theorem on Canonical Systems
}

\author{
Keshav Raj Acharya \\ Department of Mathematics, Southern Polytechnic State University, 1100 South Marietta Pkwy, Marietta, GA 30060, USA \\ Correspondence should be addressed to Keshav Raj Acharya; kacharya@spsu.edu
}

Received 21 December 2013; Accepted 18 March 2014; Published 3 April 2014

Academic Editors: G. L. Karakostas and C. Zhu

Copyright (C) 2014 Keshav Raj Acharya. This is an open access article distributed under the Creative Commons Attribution License, which permits unrestricted use, distribution, and reproduction in any medium, provided the original work is properly cited.

\begin{abstract}
The aim of this paper is to show that, in the limit circle case, the defect index of a symmetric relation induced by canonical systems, is constant on $\mathbb{C}$. This provides an alternative proof of the De Branges theorem that the canonical systems with $\operatorname{tr} H 1 \mathrm{imply}$ the limit point case. To this end, we discuss the spectral theory of a linear relation induced by a canonical system.
\end{abstract}

\section{Introduction}

This paper deals with the canonical systems of the following form:

$$
J u^{\prime}(x)=z H(x) u(x), \quad z \in \mathbb{C} .
$$

Here $J=\left(\begin{array}{cc}0 & -1 \\ 1 & 0\end{array}\right)$ and $H(x)$ is a $2 \times 2$ positive semidefinite matrix whose entries are locally integrable. For fixed $z \in \mathbb{C}$, a function $u(\cdot, z):[0, N] \rightarrow \mathbb{C}^{2}$ is called a solution if $u$ is absolutely continuous and satisfies (1). Consider the Hilbert space as follows:

$$
L^{2}\left(H, \mathbb{R}_{+}\right)=\left\{f(x, z)=\left(\begin{array}{l}
f_{1}(x) \\
f_{2}(x)
\end{array}\right):\|f\|<\infty\right\},
$$

provided with an inner product $\langle f, g\rangle=\int_{0}^{\infty} f(x)^{*} H(x) g(x) d x$.

The canonical systems ( 1$)$ on $L^{2}\left(H, \mathbb{R}_{+}\right)$have been studied by Hassi et al., Winkler, and Remling in [1-4] in various contexts. The Jacobi and Schrödinger equations can be written into canonical systems with appropriate choice of $H(x)$. In addition, canonical systems are closely connected with the theory of the De Branges spaces and the inverse spectral theory of one-dimensional Schrödinger operators; see [3]. We believe that the extensions of the theories from these equations to the canonical systems are to be of general interest.

If the system (1) can be written in the form of

$$
H(x)^{-1} J u^{\prime}=z u,
$$

then we may consider this as an eigenvalue equation of an operator on $L^{2}\left(H, R_{+}\right)$. But $H(x)$ is not invertible in general. Instead, the system (1) induces a linear relation that may have a multivalued part. Therefore, we consider this as an eigenvalue problem of a linear relation induced by (1) on $L^{2}\left(H, R_{+}\right)$.

For some $z \in \mathbb{C}$, if the canonical system (1) has all solutions in $L^{2}\left(H, \mathbb{R}_{+}\right)$, we say that the system is in the limit circle case, and if the system has unique solution in $L^{2}\left(H, \mathbb{R}_{+}\right)$, we say that the system is in limit point case. The basic results in this paper are the following theorems.

Theorem 1. In the limit circle case, the defect index $\beta\left(\mathscr{R}_{0}\right)$ of the symmetric relation $\mathscr{R}_{0}$, induced by (1), is constant on $\mathbb{C}$.

The immediate consequence of the Theorem 1 is the following theorem.

Theorem 2 (De Branges). The canonical systems with tr $H \equiv$ 1 prevail the limit point case.

Theorem 2 has been proved in [5] by function theoretic approach. However the proof was not easily readable to me and we thought of providing an alternate and simple proof of the theorem.

In order to prove the main theorems we use the results from the papers $[1,3,4]$ and we use the spectral theory of a linear relation from [6]. 
Let $\mathscr{H}$ be a Hilbert space over $\mathbb{C}$ and denote by $\mathscr{H}^{2}$ the Hilbert space $\mathscr{H} \oplus \mathscr{H}$. A linear relation $\mathscr{R}=\{(f, g): f, g \in$ $\mathscr{H}\}$ on $\mathscr{H}$ is a subspace of $\mathscr{H}^{2}$. The adjoint of $\mathscr{R}$ on $\mathscr{H}$ is a closed linear relation defined by

$$
\mathscr{R}^{*}=\left\{(h, k) \in \mathscr{H}^{2}:\langle g, h\rangle=\langle f, k\rangle \forall(f, g) \in \mathscr{R}\right\} .
$$

A linear relation $\mathcal{S}$ is called symmetric if $\mathcal{S} \subset \mathcal{S}^{*}$ and selfadjoint if $\mathcal{S}=\mathcal{S}^{*}$. The theory of such relations can be found in [5-8]. The regularity domain of $\mathscr{R}$ is the following set:

$$
\begin{gathered}
\Gamma(\mathscr{R})=\{z \in \mathbb{C}: \exists C(z)>0 \text { such that }\|(z f-g)\| \\
\geq C(z)\|f\| \forall(f, g) \in \mathscr{R}\} .
\end{gathered}
$$

The following theorem has been derived from [6].

Theorem 3. Suppose $\mathscr{T}$ is a self-adjoint relation and suppose $z \in \Gamma(\mathscr{T})$; then

$$
\mathscr{H}=\{z f-g:(f, g) \in \mathscr{T}\}
$$

The defect index $\beta(\mathscr{R}, z)$ is the dimension of defect space:

$$
R(z-\mathscr{R})^{\perp}=\{z f-g:(f, g) \in \mathscr{R}\}^{\perp}
$$

It has been shown in [6] that the defect index $\beta(\mathscr{R}, z)$ is constant on each connected subset of $\Gamma(\mathscr{R})$. Moreover, if $\mathscr{R}$ is symmetric, then the defect index is constant in the upper and lower half-planes. In addition, it is worth mentioning here the following theorem from [6] which provides us with the condition for a symmetric relation on a Hilbert space to have self-adjoint extension.

Theorem 4. (1) A symmetric relation $\mathscr{R}$ possesses self-adjoint extension if and only if $\beta(\mathscr{R}, z)$ on lower and upper half-planes is equal.

(2) A symmetric extension $\mathscr{R}^{\prime}$ of $\mathscr{R}$ is self-adjoint if and only if $\mathscr{R}^{\prime}$ is an $\beta(\mathscr{R}, z)$-dimensional extension of $\mathscr{R}$. set:

The resolvent set for a closed relation $\mathscr{R}$ is the following

$$
\begin{array}{r}
\rho(\mathscr{R})=\{z \in \mathbb{C}: \exists T \in B(\mathscr{H}) \text { such that } \\
\mathscr{R}=\{(T f, z T f-f): f \in \mathscr{H}\}\},
\end{array}
$$

and the spectrum of $\mathscr{R}$ is $\sigma(\mathscr{R})=\mathbb{C}-\rho(\mathscr{R})$.

We call $S(\mathscr{R})=\mathbb{C}-\Gamma(\mathscr{R})$ the spectral kernel of $\mathscr{R}$. The following theorem from [6] shows the relation between the spectral kernel and spectrum of a self-adjoint relation.

Theorem 5. If $\mathscr{T}$ is a self-adjoint relation and $T=(\mathscr{T}-z)^{-1}$, $z \in \Gamma(\mathscr{T})$ then one has the following

(1) $S(\mathscr{T})=\sigma(\mathscr{T})$.

(2) If $\lambda \in S(\mathscr{T})$, then $1 /(z-\lambda) \in S(T)$.

(3) $S(T) \subset \sigma(T)$.

In the next section we discuss the linear relation induced by a canonical system and prove our main theorems.

\section{Relation Induced by a Canonical System on $L^{2}\left(H, \mathbb{R}_{+}\right)$and Proof of the Main Theorems}

Consider that a relation $\mathscr{R}$ in the Hilbert space $L^{2}\left(H, \mathbb{R}_{+}\right)$is induced by (1) as

$$
\mathscr{R}=\left\{(f, g) \in\left(L^{2}\left(H, \mathbb{R}_{+}\right)\right)^{2}: f \in A C, J f^{\prime}=H g\right\},
$$

and is called the maximal relation. This relation is made up of pairs of equivalence classes $(f, g)$, such that there exists a locally absolutely continuous representative of $f$ again denoted by $f$ and a representative of $g$, again denoted by $g$, such that $J f^{\prime}=H g$ a.e. on $\mathbb{R}_{+}$. The adjoint relation $\mathscr{R}_{0}=\mathscr{R}^{*}$ is defined by

$$
\begin{gathered}
\mathscr{R}_{0}=\left\{(f, g) \in\left(L^{2}\left(H, \mathbb{R}_{+}\right)\right)^{2}:\langle g, h\rangle\right. \\
=\langle f, k\rangle \forall(h, k) \in \mathscr{R}\},
\end{gathered}
$$

and is called the minimal relation. It has been shown in [1] that $\mathscr{R}_{0}$ is close and symmetric. Moreover, $\mathscr{R}_{0} \subset \mathscr{R}$ and $\left(\mathscr{R}_{0}\right)^{*}=\mathscr{R}$.

Lemma 6 (see [1]). For each $c, d \in \mathbb{C}^{2}$ there exists $\left(\phi_{0}, \psi_{0}\right)$, $\left(\phi_{N}, \psi_{N}\right) \in \mathscr{R}$ such that $\phi_{0}, \phi_{N}$ have compact support and $\phi(0+)=c, \phi(N-)=d$.

Lemma 7 (see [1]). Let $(f, g),(h, k) \in \mathscr{R}$. Then the following limit exists:

$$
\lim _{x \rightarrow \infty} h(x) J f(x)=h(0+) J f(0+)-[\langle f, k\rangle-\langle g, h\rangle] .
$$

Lemma 8. The minimal relation $\mathscr{R}_{0}$ is given by

$$
\begin{gathered}
\mathscr{R}_{0}=\left\{(f, g) \in \mathscr{R}: f(0+)=0, \lim _{x \rightarrow \infty} f^{*}(x) \operatorname{Jh}(x)\right. \\
=0 \forall(h, k) \in \mathscr{R}\} .
\end{gathered}
$$

Proof. By Lemma 7, we get

$$
\begin{aligned}
& \left\{(f, g) \in \mathscr{R}: f(0+)=0, \lim _{x \rightarrow \infty} f^{*}(x) J h(x)\right. \\
& =0 \forall(h, k) \in \mathscr{R}\} \subset \mathscr{R}_{0} .
\end{aligned}
$$

On the other hand, let $(f, g) \in R_{0}$. By Lemma 6 for any $u \in \mathbb{C}^{2}$ there exists $(\phi, \psi) \in \mathscr{R}$ such that $\phi$ has compact support and $\phi(0+)=c$. So

$$
\begin{aligned}
0 & =\langle f, \psi\rangle-\langle g, \phi\rangle \\
& =\lim _{x \rightarrow \infty} f^{*}(x) J \phi(x)-\phi(0+) J f(0+) \\
& =u J f(0+) .
\end{aligned}
$$

This implies that $f(0+)=0$. This would also force the following:

$$
\lim _{x \rightarrow \infty} f^{*}(x) J h(x)=0 \quad \forall(h, k) \in \mathscr{R} .
$$


Note that the dimension of the solution space of the system (1) is two.

Remark 9. The defect index $\beta\left(\mathscr{R}_{0}\right)$ of the minimal relation $\mathscr{R}_{0}$ is equal to the number of linearly independent solutions of the system (1) whose class lies in $L^{2}\left(H, \mathbb{R}_{+}\right)$. Therefore, in the limit circle case, the defect indices of $\mathscr{R}_{0}$ are $(2,2)$.

Since $\mathscr{R}_{0}$ has equal defect indices, by Theorem 4 , it has self-adjoint extensions say $\mathscr{T}$. Consider a relation as follows:

$$
\begin{aligned}
\mathscr{T}^{\alpha, \beta}=\left\{(f, g) \in \mathscr{R}: f_{1}(0) \sin \alpha+f_{2}(0) \cos \alpha=0,\right. & \\
& \left.f_{1}(N) \sin \beta+f_{2}(N) \cos \beta=0, \alpha, \beta \in(0, \pi]\right\},
\end{aligned}
$$

on a compact interval $[0, N]$.

Lemma 10. $\mathscr{T}^{\alpha, \beta}$ is a self-adjoint relation.

Proof. Clearly $\mathscr{T}^{\alpha, \beta}$ is a symmetric relation because of the boundary conditions at 0 and $N$. We will show that $\mathscr{T}^{\alpha, \beta}$ is a 2-dimensional extension of $\mathscr{R}_{0}$. Then by Theorem $4, \mathscr{T}^{\alpha, \beta}$ is a self-adjoint relation. By Lemma 6 , for $c=\left(\begin{array}{c}-\cos \alpha \\ \sin \alpha\end{array}\right)$ and $w=\left(\begin{array}{c}-\cos \beta \\ \sin \beta\end{array}\right) \in \mathbb{C}^{2}$, there exist $\phi_{0}$ and $\phi_{N}$ in $D(\mathscr{R})$ such that $\phi_{0}(0+)=c$ and $\phi_{N}(N-)=w$ and the support of $\phi_{0}$ and $\phi_{N}$ is contained in $[0, N]$. Clearly $\phi_{0}$ and $\phi_{N}$ are linearly independent but $\phi_{0}$ and, $\phi_{N}$ are not in $D\left(\mathscr{R}_{0}\right)$. This shows that $D\left(\mathscr{R}_{0}\right) \subset D\left(R_{0}\right)+L\left(\phi_{0}, \phi_{N}\right) \subset D\left(\mathscr{T}^{\alpha, \beta}\right)$. Because of the boundary condition at 0 and $N, D\left(\mathscr{T}^{\alpha, \beta}\right)$ is 2-dimensional restriction of $D(\mathscr{R})$. Hence $D\left(\mathscr{T}^{\alpha, \beta}\right)=D\left(\mathscr{R}_{0}\right)+L\left(\phi_{0}, \phi_{N}\right)$. Therefore, $\mathscr{T}^{\alpha, \beta}$ is 2 -dimensional extension of $\mathscr{R}_{0}$ so that $\mathscr{T}^{\alpha, \beta}$ is a self-adjoint relation.

Let $u(x, z)$ and $v(x, z)$ be the solution of the canonical system $(1)$ on $[0, N]$ with the initial values:

$$
u(0, z)=\left(\begin{array}{l}
1 \\
0
\end{array}\right), \quad v(0, z)=\left(\begin{array}{l}
0 \\
1
\end{array}\right) .
$$

For $z \in C^{+}$there is a unique $m(z)$ such that $f(x, z)=u(x, z)+$ $m(z) v(x, z)$ satisfying the following:

$$
f_{1}(N, z) \sin \beta+f_{2}(N, z) \cos \beta=0 .
$$

This is well defined because $u$ does not satisfy the boundary condition at $N$; otherwise, $z$ will be an eigenvalue of some self-adjoint relation $\mathscr{T}^{\alpha, \beta}$.

Next, we describe the spectrum of $\mathscr{T}^{\alpha, \beta}$. Let

$$
T(x, z)=\left(\begin{array}{ll}
u_{1}(x, z) & v_{1}(x, z) \\
u_{2}(x, z) & v_{2}(x, z)
\end{array}\right), \quad T(0, z)=\left(\begin{array}{ll}
1 & 0 \\
0 & 1
\end{array}\right),
$$

and define

$$
w_{\alpha}(x, z)=\frac{1}{\sin \alpha+m(z) \cos \alpha} T(x, z)\left(\begin{array}{c}
\cos \alpha \\
-\sin \alpha
\end{array}\right) .
$$

It is not hard to see that.
Lemma 11. Using the notation above one has

$$
\begin{gathered}
f(x, z) w_{\alpha}(x, \bar{z})^{*}-w_{\alpha}(x, z) f(x, \bar{z})^{*} \\
=T(x, z) J T(x, \bar{z})^{*}=J .
\end{gathered}
$$

Lemma 12. Let $z \in \Gamma\left(\mathscr{T}^{\alpha, \beta}\right)$; then $\left(\mathscr{T}^{\alpha, \beta}-z\right)^{-1}$ is a bounded linear operator and is defined by

$$
\left(\mathscr{T}^{\alpha, \beta}-z\right)^{-1} h(x)=\int_{0}^{N} G(x, t, z) H(t) h(t) d t,
$$

where

$$
G(x, t, z)= \begin{cases}f(x, z) w_{\alpha}\left(t, \bar{z}_{0}\right)^{*} & \text { if } 0<t \leq x \\ w_{\alpha}(t, \bar{z}) f\left(x, \bar{z}_{0}\right) & \text { if } x<t \leq N .\end{cases}
$$

Proof. Let $y(x, z)=\int_{0}^{N} G(x, t, z) H(t) h(t) d t$. We show that $y(x, z)$ solves the inhomogeneous equation as follows:

$$
J y^{\prime}=z H y-H h,
$$

for a.e. $x>0$. Here

$$
\begin{aligned}
y(x, z)= & \int_{0}^{x} f(x, z) w_{\alpha}(t, \bar{z})^{*} H(t) h(t) d t \\
& +\int_{x}^{N} w_{\alpha}(x, z) f(t, \bar{z})^{*} H(t) h(t) d t
\end{aligned}
$$

and $J f^{\prime}=z H f, J w_{\alpha}^{\prime}=z H w_{\alpha}$. Then on differentiation we get

$$
\begin{aligned}
y^{\prime}(x, z)= & f(x, z) w_{\alpha}(x, \bar{z})^{*} H(x) h(x) \\
& +f^{\prime}(x, z) \int_{0}^{x} w_{\alpha}(t, \bar{z})^{*} H(t) h(t) d t \\
& -w_{\alpha}(x, z) f(x, \bar{z})^{*} H(x) h(x) \\
& +w_{\alpha}^{\prime}(x, z) \int_{x}^{N} f(t, \bar{z})^{*} H(t) h(t) d t .
\end{aligned}
$$

Then

$$
\begin{aligned}
J y^{\prime}(x, z)= & J f(x, z) w_{\alpha}(x, \bar{z})^{*} H(x) h(x) \\
& +J f^{\prime}(x, z) \int_{0}^{x} w_{\alpha}(t, \bar{z})^{*} H(t) h(t) d t \\
& -J w_{\alpha}(x, z) f(x, \bar{z})^{*} H(x) h(x) \\
& +J w_{\alpha}^{\prime}(x, z) \int_{x}^{N} f(t, \bar{z})^{*} H(t) h(t) d t \\
= & J f(x, z) w_{\alpha}(x, \bar{z})^{*} H(x) h(x) \\
& +z H f(x, z) \int_{0}^{x} w_{\alpha}(t, \bar{z})^{*} H(t) h(t) d t \\
& -J w_{\alpha}(x, z) f(x, \bar{z})^{*} H(x) h(x) \\
& +z H w_{\alpha}(x, z) \int_{x}^{N} f(t, \bar{z})^{*} H(t) h(t) d t
\end{aligned}
$$




$$
\begin{aligned}
& =J\left(f(x, z) w_{\alpha}(x, \bar{z})^{*}-w_{\alpha}(x, z) f(x, \bar{z})^{*}\right) H h \\
& +z H\left(\int_{0}^{x} f(x, z) w_{\alpha}(t, \bar{z})^{*} H(t) h(t) d t\right. \\
& \left.\quad+\int_{x}^{N} w_{\alpha}(x, z) f(t, \bar{z})^{*} H(t) h(t) d t\right) \\
& =J J H h+z H y \\
& =z H y-H h .
\end{aligned}
$$

On the other hand denote $g(x, z)$ as

$$
g(x, z)=\left(\mathscr{T}^{\alpha, \beta}-z\right)^{-1} h(x)
$$

then by Theorem 3, $h(x)=z u-v$ for some $(u, v) \in$ $\mathscr{T}^{\alpha, \beta}$ so that $(g, z g-h) \in \mathscr{T}^{\alpha, \beta}$. So $g(x, z)$ also satisfies the inhomogeneous problem and $g(x, z) \in D\left(\mathscr{T}^{\alpha, \beta}\right)$; it satisfies the boundary condition which implies that $g(0, z)=$ $\left(\begin{array}{c}\cos \alpha \\ -\sin \alpha\end{array}\right) c(z)$ for some scalar $c(z)$. We have

$$
y(0, z)=\frac{1}{\sin \alpha+m(z) \cos \alpha}\left(\begin{array}{c}
\cos \alpha \\
-\sin \alpha
\end{array}\right)\langle f(x, \bar{z}), h\rangle .
$$

Now

$$
\begin{aligned}
\langle f(\cdot, \bar{z}), h\rangle= & \langle f(\cdot, \bar{z}), h\rangle-\langle f(\cdot, \bar{z}), z g\rangle+\langle f(\cdot, \bar{z}), z g\rangle \\
= & \langle f(\cdot, \bar{z}), h-z g\rangle+z\langle f(\cdot, \bar{z}), g\rangle \\
= & -\int_{0}^{N} f(x, \bar{z})^{*} H(z g-h) d x \\
& +z \int_{0}^{N} f(x, \bar{z})^{*} H g d x \\
= & -\int_{0}^{N} f(x, \bar{z})^{*} J g^{\prime} d x-\int_{0}^{N} f^{\prime}(x, \bar{z})^{*} J g d x \\
= & f(0, \bar{z})^{*} J g(0, z)-f(N, \bar{z})^{*} J g(N, z) .
\end{aligned}
$$

Since both $f(x, z)$ and $g(x, z)$ satisfy the same boundary condition at $N f(N, \bar{z})^{*} J g(N, z)=0$, now

$$
f(0, \bar{z})^{*} J g(0, z)=(1, m(z))\left(\begin{array}{cc}
0 & -1 \\
1 & 0
\end{array}\right)\left(\begin{array}{c}
\cos \alpha \\
-\sin \alpha
\end{array}\right) c(z) .
$$

So

$$
\begin{aligned}
y(0, z)= & \frac{1}{\sin \alpha+m(z) \cos \alpha}\left(\begin{array}{c}
\cos \alpha \\
-\sin \alpha
\end{array}\right)(1, m(z)) \\
& \times\left(\begin{array}{cc}
0 & -1 \\
1 & 0
\end{array}\right)\left(\begin{array}{c}
\cos \alpha \\
-\sin \alpha
\end{array}\right) c(z) \\
= & \frac{1}{\sin \alpha+m(z) \cos \alpha}\left(\begin{array}{c}
\cos \alpha \\
-\sin \alpha
\end{array}\right)(m(z),-1) \\
& \times\left(\begin{array}{c}
\cos \alpha \\
-\sin \alpha
\end{array}\right) c(z) \\
= & \left(\begin{array}{c}
\cos \alpha \\
-\sin \alpha
\end{array}\right) c(z) \\
= & g(0, z) .
\end{aligned}
$$

By uniqueness we must have $g(x, z)=y(x, z)$. Moreover, $\left(\mathscr{T}^{\alpha, \beta}-z\right)^{-1}$ is a bounded linear operator.

Now define a map $V: L^{2}(H,[0, N]) \rightarrow L^{2}(I,[0, N])$ by

$$
V y=H^{1 / 2}(x) y(x) .
$$

Here $H^{1 / 2}(x)$ is the unique positive semidefinite square root of $H(x)$. Then $V$ is an isometry and hence maps $L^{2}(H,[0, N])$ unitarily onto the range $R(V) \subset L^{2}(I,[0, N])$. Define an integral operator $\mathscr{L}$ on $L^{2}(I,[0, N])$ as

$$
(\mathscr{L} f)(x)=\int_{0}^{N} L(x, t) f(t) d t
$$

$$
L(x, t)=H^{1 / 2}(x) G(x, t) H^{1 / 2}(t) .
$$

The kernel $L$ is square integrable since

$$
\begin{aligned}
\iint_{0}^{N}\left\|L^{*} L\right\| d x d t & \leq \iint_{0}^{N}\left\|V G^{*}\right\|\left\|(V G)^{*}\right\| d x d t \\
& \leq \iint_{0}^{N}\left\|G^{*}\right\|\|G\| d x d t<\infty .
\end{aligned}
$$

So $\mathscr{L}$ is a Hilbert-Schmidt operator and thus compact. Notice that $L(x, t)=L^{*}(t, x)$. This implies that $\mathscr{L}$ is self-adjoint.

Lemma 13 (see [3]). Let $f \in L^{2}(I,[0, N]), \lambda \neq 0$, and then the following statements are equivalent.

(1) $\mathscr{L} f=\lambda^{-1} f$.

(2) $f \in R(V)$, and the unique $y \in L^{2}(H,[0, N])$ with $V y=$ $f$ solves $\left(\mathscr{T}^{\alpha, \beta}-z\right)^{-1} y=\lambda y$.

Proof. For all $g \in L^{2}(I,[0, N])$ we have

$$
\begin{aligned}
(\mathscr{L} g)(x) & =H^{1 / 2}(x) w(x) \quad \text { where } w(x) \\
& =\int_{0}^{N} G(x, t) H^{1 / 2}(t) g(t) d t
\end{aligned}
$$


lies in $L^{2}(H,[0, N])$. Then $R(\mathscr{L}) \subset R(V)$. Now if (1) holds, then $f=\lambda \mathscr{L} f \in R(V)$. So $f=V(y)$ for unique $y \in$ $L^{2}(H,[0, N])$ and

$$
\begin{aligned}
f(x) & =H^{1 / 2}(x) y(x)=\lambda \mathscr{L} y(x) \\
& =\lambda H^{\frac{1}{2}}(x) \int_{0}^{N} G(x, t) H(t) y(t) d t,
\end{aligned}
$$

for a.e. $x \in[0, N]$. In other words,

$$
H^{1 / 2}(x)\left(y(x)-\lambda \int_{0}^{N} G(x, t) H(t) y(t) d t\right)=0 .
$$

Conversely if (11) holds,

$$
\lambda y=\int_{0}^{N} G(x, t) H(t) y(t) d t,
$$

And then $H^{1 / 2}(x) y=(1 / \lambda) \int_{0}^{N} H^{1 / 2}(x) G(x, t) H(t) y(t) d t$.

Lemma 14. Let $z \in \mathbb{C}$. For any $\lambda \neq z$, if $(f, \lambda f) \in \mathscr{T}^{\alpha, \beta}$, then $f$ solves $\left(\mathscr{T}^{\alpha, \beta}-z\right)^{-1} y=1 /(\lambda-z) y$. Conversely, if $y \in$ $L^{2}(H,[0, N])$ and $y$ solves $\left(T^{\alpha, \beta}-z\right)^{-1} y=\lambda y$, then $(y,(z+1 /$

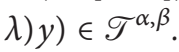

Proof. Let $(f, \lambda f) \in \mathscr{T}^{\alpha, \beta}$; then $(f, \lambda f-z f) \in\left(\mathscr{T}^{\alpha, \beta}-z\right)$. It follows that

$$
\begin{aligned}
& ((\lambda-z) f, f) \in\left(\mathscr{T}^{\alpha, \beta}-z\right)^{-1} \\
& \quad \Longrightarrow\left(f, \frac{1}{(\lambda-z)}\right) \in\left(\mathscr{T}^{\alpha, \beta}-z\right)^{-1} .
\end{aligned}
$$

This means that $f$ solves

$$
\left(\mathscr{T}^{\alpha, \beta}-z\right)^{-1} y=\frac{1}{\lambda-z} y .
$$

Conversely suppose $y \in L^{2}(H,[0, N])$ and $y$ solves

$$
\left(\mathscr{T}^{\alpha, \beta}-z\right)^{-1} y=\lambda y .
$$

That is, $(y, \lambda y) \in\left(\mathscr{T}^{\alpha, \beta}-z\right)^{-1}$ so that $(\lambda y, y) \in\left(\mathscr{T}^{\alpha, \beta}-z\right)$. So there is $(f, g) \in \mathscr{T}^{\alpha, \beta}$ such that $\lambda y=f$ and

$$
g-z f=y \Longrightarrow g=y+z \lambda y .
$$

Hence

$$
(\lambda y, y+z \lambda y) \in \mathscr{T}^{\alpha, \beta} \Longrightarrow\left(y,\left(z+\frac{1}{\lambda} y\right)\right) \in \mathscr{T}^{\alpha, \beta} .
$$

By Lemma 13, we see that there is a one-to-one correspondence of eigenvalues (eigenfunctions) for the operator $\mathscr{L}$ and $\left(\mathscr{T}^{\alpha, \beta}-z\right)^{-1}$. As $\mathscr{L}$ is compact operator, it has only discrete spectrum consisting of only eigenvalues. Since $\left(\mathscr{T}^{\alpha, \beta}-z\right)^{-1}$ is unitarily equivalent to $\mathscr{L} L_{R(V)}$; that is,

$$
V^{-1} \mathscr{L} \bigsqcup_{R(V)} V=\left(\mathscr{T}^{\alpha, \beta}-z\right)^{-1},
$$

$\left(\mathscr{T}^{\alpha, \beta}-z\right)^{-1}$ has only discrete spectrum consisting of only eigenvalues. Then, by Theorem $5, \mathscr{T}^{\alpha, \beta}$ has only discrete spectrum. By Lemma 14, the spectrum of $\mathscr{T}^{\alpha, \beta}$ consists only of eigenvalues. Hence we have

$$
\sigma\left(\mathscr{T}^{\alpha, \beta}\right)=\left\{z \in \mathbb{C}: u_{\alpha 1}(N, z) \sin \beta+u_{\alpha 2} \cos \beta=0\right\} .
$$

We would like to extend this idea over the half line $\mathbb{R}_{+}$. First note that we are considering the limit circle case of the system (1). That implies that for any $z \in \mathbb{C}^{+}$the defect indices of $\mathscr{R}_{0}$ are $(2,2)$. Suppose $p \in D(\mathscr{R}) \backslash D\left(\mathscr{R}_{0}\right)$ such that $\lim _{x \rightarrow \infty} p(x)^{*} J p(x)=0$. Such function clearly exists.

Consider the following relation:

$$
\begin{gathered}
\mathscr{T}^{\alpha, p}=\left\{(f, g) \in \mathscr{R}: f_{1}(0) \sin \alpha+f_{2}(0, z) \cos \alpha=0\right. \text { and } \\
\left.\lim _{x \rightarrow \infty} f(x)^{*} J p(x)=0\right\} .
\end{gathered}
$$

Lemma 15. $\mathscr{T}^{\alpha, p}$ defines a self-adjoint extension of $\mathscr{R}_{0}$.

Proof. Let $u(x)$ be a solution of the system (1) with some initial values and $p(x)$ as above. Define $u_{0}(x)=0$ near the neighborhood of $\infty$; that is, $\lim _{x \rightarrow \infty} u_{0}(x)=0$, and $u_{0}(x)=$ $u(x)$ otherwise. Similarly, $p_{0}(x)=0$ in the neighborhood of 0 and $p_{0}(x)=p(x)$ otherwise. Then clearly $u_{0}, p_{0} \notin D\left(\mathscr{R}_{0}\right)$ and are linearly independent. Clearly $D\left(\mathscr{R}_{0}\right)+L\left(u_{0}, p_{0}\right) \subset$ $D\left(\mathscr{T}^{\alpha, p}\right)$. Since $D\left(\mathscr{T}^{\alpha, p}\right)$ is at least 2-dimensional restriction of $D(\mathscr{R})$,

$$
D\left(\mathscr{T}^{\alpha, p}\right)=D\left(\mathscr{R}_{0}\right)+L\left(u_{0}, p_{0}\right) .
$$

Hence $\mathscr{T}^{\alpha, p}$ is a self-adjoint relation.

We next discuss the spectrum of $\mathscr{T}^{\alpha, p}$. Let $u(x, z)$ and $v(x, z)$ be two linearly independent solutions of the system (1) with

$$
u(0, z)=\left(\begin{array}{l}
1 \\
0
\end{array}\right), \quad v(0, z)=\left(\begin{array}{l}
0 \\
1
\end{array}\right)
$$

Let $z \in \mathbb{C}^{+}$and as above write $f(x, z)=u(x, z)+m(z) v(x$, $z) \in L^{2}\left(H, \mathbb{R}_{+}\right)$satisfying $\lim _{x \rightarrow \infty} f(x, z)^{*} J P(x)=0$. Let $T(x, z)=\left(\begin{array}{ll}u_{1} & v_{1} \\ u_{2} & v_{2}\end{array}\right)$ and

$$
w_{\alpha}(x, z)=\frac{1}{\sin \alpha+m(z) \cos \alpha} T(x, z)\left(\begin{array}{c}
\cos \alpha \\
-\sin \alpha
\end{array}\right) .
$$

Then as in Lemma 11 we have

$$
\begin{aligned}
f(x, z) w_{\alpha}(x, \bar{z})^{*}-w_{\alpha}(x, z) f(x, \bar{z})^{*} & =T(x, z) J T(x, \bar{z})^{*} \\
& =J .
\end{aligned}
$$


Lemma 16. Let $z \in \rho\left(\mathscr{T}^{\alpha, p}\right)$; then the resolvent operator $\left(\mathscr{T}^{\alpha, p}-z\right)^{-1}$ is given by

$$
\left(\mathscr{T}^{\alpha, p}-z\right)^{-1} h(x)=\int_{0}^{\infty} G(x, t, z) H(t) h(t) d t,
$$

where

$$
G(x, t, z)= \begin{cases}f(x, z) w_{\alpha}(t, \bar{z})^{*} & \text { if } 0<t \leq x \\ w_{\alpha}(t, \bar{z}) f(x, \bar{z}) & \text { if } x<t \leq \infty .\end{cases}
$$

Proof. Let $y(x, z)=\int_{0}^{\infty} G(x, t, z) H(t) h(t) d t$; then $y$ solves the following inhomogeneous equation:

$$
J y^{\prime}=z H y-H h .
$$

This is clear by differentiating

$$
\begin{aligned}
y(x, z)= & \int_{0}^{x} f(x, z) w_{\alpha}(t, \bar{z})^{*} H(t) h(t) d t \\
& +\int_{x}^{\infty} w_{\alpha}(x, z) f(t, \bar{z})^{*} H(t) h(t) d t .
\end{aligned}
$$

On the other hand denote $g(x, z)$ as $g(x, z)=\left(\mathscr{T}^{\alpha, p}-\right.$ $z)^{-1} h(x)$; then, by Theorem $3, h(x)=z u-v$ for some $(u, v) \epsilon$ $\mathscr{T}^{\alpha, p}$ so that $(g, z g-h) \in \mathscr{T}^{\alpha, p}$, and hence $g$ satisfies the inhomogeneous equation. Since $g \in D\left(\mathscr{T}^{\alpha, p}\right)$,

$$
\begin{gathered}
g_{1}(0, z) \sin \alpha+g_{2}(0, z) \cos \alpha=0, \\
\lim _{x \rightarrow \infty} g^{*}(x, z) \operatorname{Jp}(x, z)=0 .
\end{gathered}
$$

We also have $\lim _{x \rightarrow \infty} f^{*}(x, z) J g(x, z)=0$ and $g(0, z)=$ $\left(\begin{array}{c}\cos \alpha \\ -\sin \alpha\end{array}\right) c(z)$ for some scalar $c(z)$. But we also have

$$
y(0, z)=\frac{1}{(m(z) \cos \alpha+\sin \alpha)}\left(\begin{array}{c}
\cos \alpha \\
\sin \alpha
\end{array}\right)\langle f(\bar{z}), h\rangle .
$$

Here

$$
\begin{aligned}
\langle f(\bar{z}), h\rangle & =\langle f(\bar{z}), h\rangle-\langle f(\bar{z}), z g\rangle-\langle\bar{z} f(\bar{z}), g\rangle \\
& =\langle f(\bar{z}), h+z g\rangle-\langle\bar{z} f(\bar{z}), g\rangle \\
& =f^{*}(0, \bar{z}) J g(0, z)-\lim _{x \rightarrow \infty} f^{*}(x, z) J g(x, z) \\
& =f^{*}(0, \bar{z}) J g(0, z) .
\end{aligned}
$$

Hence $y(0, z)=g(0, z)$. By uniqueness we have $y(x, z)=$ $g(x, z)$.

Now define a map $V: L^{2}\left(H, \mathbb{R}_{+}\right) \rightarrow L^{2}\left(I, \mathbb{R}_{+}\right)$by $V y=$ $H^{1 / 2}(x) y(x) . \quad V$ is isometry and maps unitarily onto the range $R(V) \subset L^{2}\left(I, \mathbb{R}_{+}\right)$.

Define an integral operator $\mathscr{L}$ on $L^{2}\left(I, \mathbb{R}_{+}\right)$by

$$
\begin{aligned}
& (\mathscr{L} g)(x)=\int_{0}^{\infty} L(x, t) g(t) d t \\
& L(x, t)=H^{1 / 2}(x) G(x, t, z) H^{1 / 2}(t) .
\end{aligned}
$$

Then as before the kernel $L$ is square integrable. This means that

$$
\iint_{0}^{\infty}\left\|L^{*} L\right\|<\infty
$$

Hence $\mathscr{L}$ is a Hilbert Schmidt operator and so it is a compact operator. The following two lemmas are extended from the bounded interval $[0, N]$ to $\mathbb{R}_{+}$and the proofs are exactly the same as the proofs of Lemmas 13 and 14 .

Lemma 17 (see [3]). Let $f \in L^{2}\left(I, R_{+}\right), \lambda \neq 0$, and then the following statements are equivalent.

(1) $\mathscr{L} f=\lambda^{-1} f$.

(2) $f \in R(V)$, and the unique $y \in L^{2}\left(H, R_{+}\right)$with $V y=f$ solves $\left(\mathscr{T}^{\alpha, p}-z\right)^{-1} y=\lambda y$.

Lemma 18. Let $z \in \mathbb{C}$. For any $\lambda \neq z$, if $(y, \lambda y) \in \mathscr{T}^{\alpha, p}$, then $y$ solves $\left(\mathscr{T}^{\alpha, p}-z\right)^{-1} y=(1 /(\lambda-z)) y$. Conversely, if $y \in L^{2}\left(H, R_{+}\right)$and $y$ solves $\left(T^{\alpha, p}-z\right)^{-1} y=\lambda y$, then $(y,(z+$ $1 / \lambda) y) \in \mathscr{T}^{\alpha, p}$.

Again, by Lemma 17, we have a one-to-one correspondence of eigenvalues (eigenfunctions) for the operator $\mathscr{L}$ and $\left(\mathscr{T}^{\alpha, p}-z\right)^{-1}$. As $\mathscr{L}$ is compact operator, it has only discrete spectrum consisting of only eigenvalues and possibly zero. Since $\left(\mathscr{T}^{\alpha, p}-z\right)^{-1}$ is unitarily equivalent to $\mathscr{L} L_{R(V)}$; that is, $V^{-1} \mathscr{L} L_{R(V)} V=\left(\mathscr{T}^{\alpha, p}-z\right)^{-1},\left(\mathscr{T}^{\alpha, p}-z\right)^{-1}$ has only discrete spectrum consisting of only eigenvalues. Then, by Theorem 5 , $\mathscr{T}^{\alpha, p}$ has only discrete spectrum. By Lemma 18 the spectrum of $\mathscr{T}^{\alpha, p}$ consists of only eigenvalues.

With these theories in hand, we are now ready to prove the main theorems.

Proof of Theorem 1. Since $\mathscr{R}_{0}$ is a symmetric relation, the defect index $\beta\left(\mathscr{R}_{0}, z\right)$ is constant on upper and lower halfplanes. In the limit circle case, if $z$ is in upper or lower halfplanes, $\beta\left(\mathscr{R}_{0}, z\right)=2$. Suppose $\beta\left(\mathscr{R}_{0}, \lambda\right)<2$ for some $\lambda \in \mathbb{R}$. Since $\Gamma\left(\mathscr{R}_{0}\right)$ is open, $\lambda \notin \Gamma\left(\mathscr{R}_{0}\right)$, and hence $\lambda \in S\left(\mathscr{R}_{0}\right)$. Since, for each $\alpha \in(0, \pi], \mathscr{T}^{\alpha, p}$ is self-adjoint extension of $\mathscr{R}_{0}, \lambda \in S\left(\mathscr{T}^{\alpha, p}\right)=\sigma\left(\mathscr{T}^{\alpha, p}\right)$. In the limit circle case, $\sigma\left(\mathscr{T}^{\alpha, p}\right)$ consists of only eigenvalues. Therefore, $\lambda$ is an eigenvalue for all boundary conditions $\alpha$ at 0 . However, this is impossible unless $\beta\left(\mathscr{R}_{0}, \lambda\right)=2$.

Proof of Theorem 2. Suppose it prevails the limit circle case. By Theorem 1 , the defect index $\beta\left(\mathscr{R}_{0}, z\right)=\operatorname{dim} N(\mathscr{R}, \bar{z})=2$ for all $z \in \mathbb{C}$. In other words, for any $z \in \mathbb{C}$, all solutions of (1) are in $L^{2}\left(H, \mathbb{R}_{+}\right)$. In particular, the constant solutions $u(x)=$ $\left(\begin{array}{l}1 \\ 0\end{array}\right)$ and $v(x)=\left(\begin{array}{l}0 \\ 1\end{array}\right)$ of (1) when $z=0$ are in $L^{2}\left(H, \mathbb{R}_{+}\right)$. But this is not possible because $\int_{0}^{\infty} u(x)^{*} H(x) u(x) d x+$ $\int_{0}^{\infty} v(x)^{*} H(x) v(x) d x=\int_{0}^{\infty} \operatorname{tr} H(x) d x=\infty$.

\section{Conflict of Interests}

The author declares that there is no conflict of interests regarding the publication of this paper. 


\section{References}

[1] S. Hassi, H. De Snoo, and H. Winkler, "Boundary-value problems for two-dimensional canonical systems," Integral Equations and Operator Theory, vol. 36, no. 4, pp. 445-479, 2000.

[2] S. Hassi, C. Remling, and H. de Snoo, "Subordinate solutions and spectral measures of canonical systems," Integral Equations and Operator Theory, vol. 37, no. 1, pp. 48-63, 2000.

[3] C. Remling, "Schrödinger operators and de Branges spaces," Journal of Functional Analysis, vol. 196, no. 2, pp. 323-394, 2002.

[4] H. Winkler, "The inverse spectral problem for canonical systems," Integral Equations and Operator Theory, vol. 22, no. 3, pp. 360-374, 1995.

[5] L. de Branges, "Some Hilbert spaces of entire functions. II," Transactions of the American Mathematical Society, vol. 99, pp. 118-152, 1961.

[6] K. R. Acharya, "Self-adjoint extension and spectral theory of a linear relation in a Hilbert space," ISRN Mathematical Analysis, vol. 2014, Article ID 471640, 5 pages, 2014.

[7] E. A. Coddington, Extension Theory of Formally Normal and Symmetric Subspaces, vol. 134 of Memoirs, American Mathematical Society, Providence, RI, USA, 1973.

[8] A. Dijksma and H. S. V. de Snoo, "Self-adjoint extensions of symmetric subspaces," Pacific Journal of Mathematics, vol. 54, pp. 71-100, 1974. 




Advances in

Operations Research

mansans

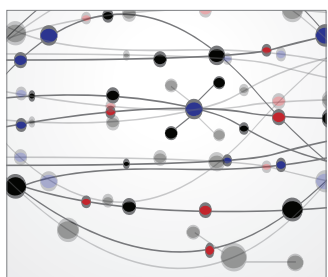

The Scientific World Journal
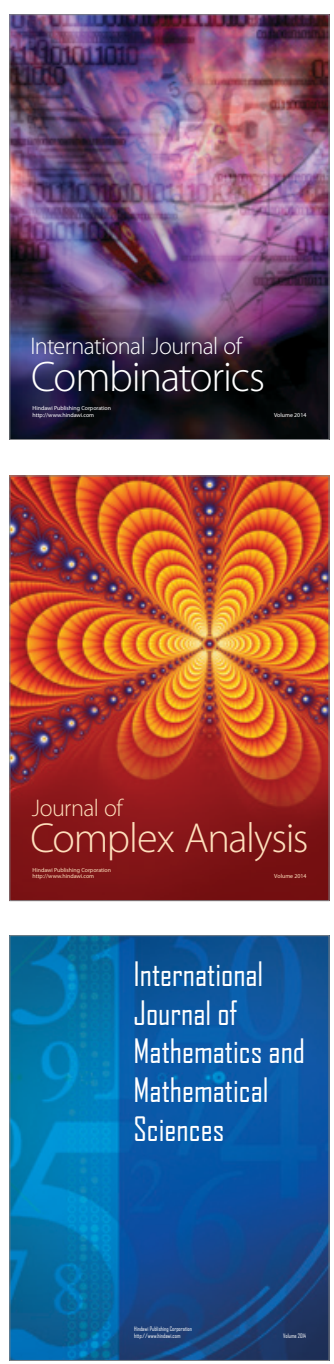


Submit your manuscripts at http://www.hindawi.com
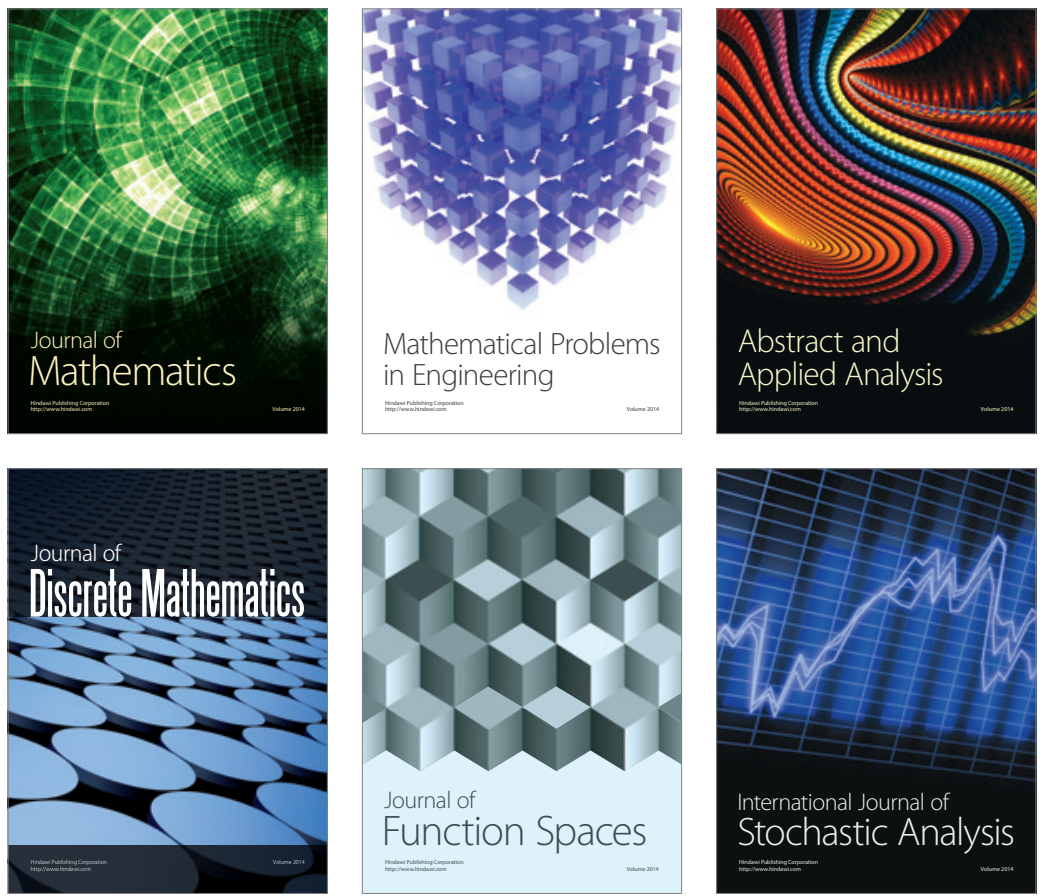

Journal of

Function Spaces



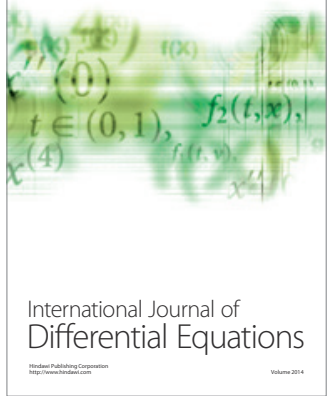
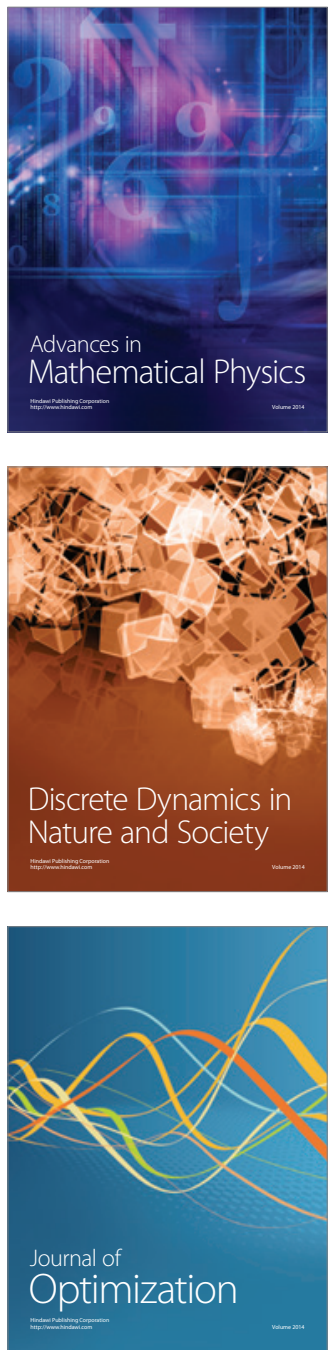